\title{
High Voltage Coil Current Sensor for DC-DC Converters Employing DDCC
}

\author{
Martin DRINOVSKY ${ }^{1,2}$, Jiri HOSPODKA ${ }^{1}$ \\ ${ }^{1}$ Dept. of Circuit Theory, Czech Technical University in Prague, Technická 2, 16627 Praha, Czech Republic \\ ${ }^{2}$ STMicroelectronics Design and Application s.r.o., Pobřežní 620/3, 18600 Praha, Czech Republic
}

martin.drinovsky@st.com, hospodka@fel.cvut.cz

\begin{abstract}
Current sensor is an integral part of every switching converter. It is used for over-current protection, regulation and in case of multiphase converters for balancing. A new high voltage current sensor for coil-based current sensing in DC-DC converters is presented. The sensor employs DDCC with high voltage input stage and gain trimming. The circuit has been simulated and implemented in $0.35 \mu \mathrm{m}$ $B C D$ technology as part of a multiphase DC-DC converter where its function has been verified. The circuit is able to sustain common mode voltage on the input up to $40 \mathrm{~V}$, it occupies $0.387 \times 0.345 \mathrm{~mm}^{2}$ and consumes $3.2 \mathrm{~mW}$ typically.
\end{abstract}

\section{Keywords}

Current sensor, current conveyor, DDCC, DC-DC converter

\section{Introduction}

The switching converters have become an important part of nearly every electronic system, such as mobile phones, tablets or computers. One of the widely used switching converter topology is the buck converter used for converting high input voltage into a lower output voltage. The buck converter uses a coil to transfer current from the input to the output. This current is often measured for various purposes, e.g. for over-current protection or for regulation when a current control loop is employed.

Several approaches for current sensing exist [1-2]. When the power MOS transistors are integrated on-chip the copy or sense MOS technique is used [3-5]. For applications with off chip power transistors other techniques measuring coil current must be used.

One way of measuring coil current uses a sensing resistor in series with the coil as depicted in Fig. 1a. This has the disadvantage of introducing additional loss into the system and lowering overall efficiency. Other method uses the parasitic resistance of the coil (DCR) to measure the current [1]. This is done by adding a filter in parallel to the coil as shown in Fig. $1 \mathrm{~b}$, where $R_{L}$ represents a DC resistance of the coil. If the following condition holds

$$
R C=\frac{L}{R_{L}},
$$

then the voltage on the capacitor is proportional to the inductor current as

$$
v_{C}(t)=R_{L} i_{L}(t) .
$$

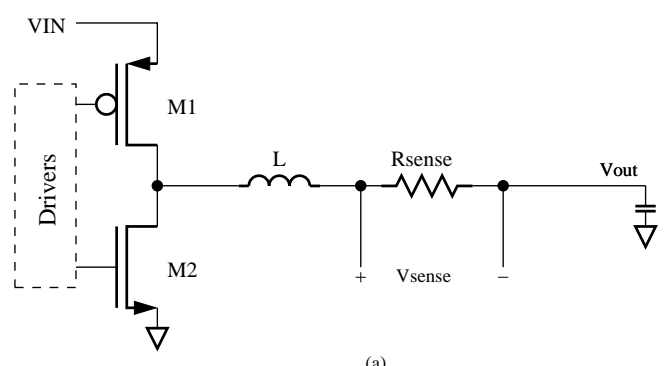

(a)

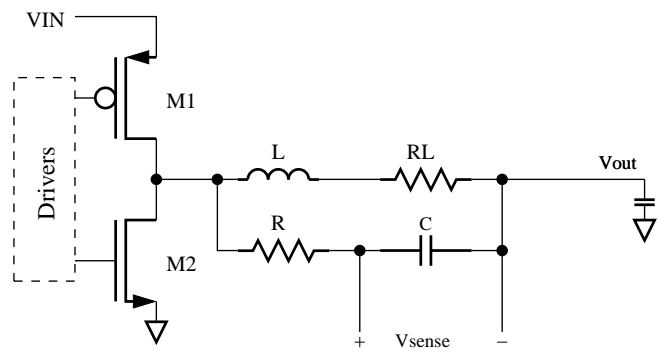

(b)

Fig. 1. Coil current sensing: (a) On external resistor and (b) filter-based sensing on coil resistance.

One of the circuits sensing coil current is presented in [6], this solution cannot be used for high voltage sensing as it is limited by the capability of the standard voltage components. The sensing circuit presented in [7] requires an operational amplifier with both high voltage input and output. This complicates design because all the basic structures must be cascoded with high voltage components. The same principle is also used in [8], where the current sensors uses the current shunt monitor chip INA139. The circuit in [9] uses instrumentation amplifier to sense the coil current, however it still requires high voltage operational amplifiers. Another solution [10] uses capacitors to sample the input voltage but it requires capacitors with high voltage capability available in the given process.

In this paper a novel current sensor with high voltage input is presented. It is based around a differential-difference current conveyor (DDCC) with only one input stage with 


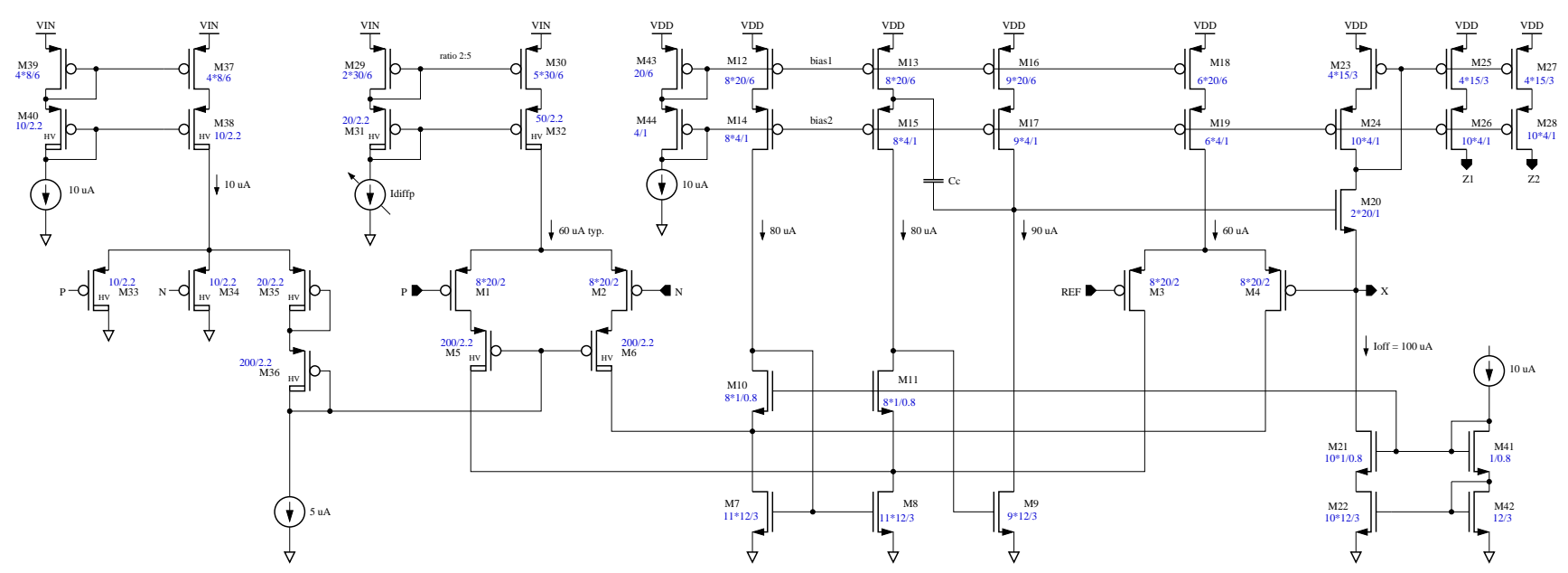

Fig. 2. Schematic of the proposed DDCC based current sensor (dimension are in $\mu \mathrm{m}$ ).

high voltage requirements thereby reducing the number of high voltage components, area and design effort. The circuit description is presented in Sec. 2, achieved results can be found in Sec. 3 and conclusion follows in Sec. 4.

\section{Proposed Circuit}

The block diagram of the proposed current sensor is shown in Fig. 3. The basic building block is the DDCC [11] with multiple outputs which is described in ideal form as

$$
\begin{aligned}
I_{\mathrm{REF}} & =0, I_{P}=0, I_{N}=0, \\
V_{X} & =V_{\mathrm{REF}}+\alpha\left(V_{P}-V_{N}\right), \\
I_{Z 1} & =I_{Z 2}=I_{X}-I_{\mathrm{off}},
\end{aligned}
$$

where $I_{\text {off }}$ is internally added offset current to allow simple bidirectional signal processing by the subsequent circuitry and the gain $\alpha$ equals 1 for a traditional DDCC. The detailed analysis of the DDCC behaviour has been carried in [11]. For accurate $\alpha$ the internal feedback loop must have a sufficient gain, this is guaranteed by cascoding of the current mirrors. Another major source of inaccuracy are the nonlinearities of the transconductance stages. These lead to complex expressions with little insight, however, for the given application they are tolerable as will be shown in the simulation results.

The sensed voltage difference on the $R_{\text {sense }}$ is copied by the DDCC onto a resistor $R_{\mathrm{copy}}$, therefore the output currents are proportional to the coil current as

$$
I_{Z 1,2}=\alpha I_{L} \frac{R_{\text {sense }}}{R_{\text {copy }}}-I_{\text {off }} .
$$

The output currents are identical and can be easily added and scaled according to the various needs of the application. The $V_{\text {bias }}$ is a constant inaccurate voltage selected in the common mode range of the inputs. To save the application board space and reduce component count the resistor $R_{\text {copy }}$ is implemented on-chip. However, the technological spread of the resistor leads to the inaccuracy of the gain. This is compensated by trimming the gain $\alpha$ as will be described further.

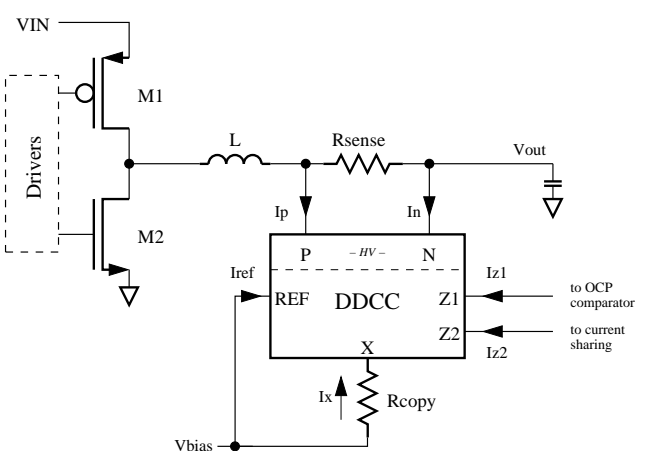

Fig. 3. Block diagram of the proposed DDCC based current sensor.

The schematic of the proposed DDCC can be found in Fig. 2. The circuit employs two

3.3 V differential pairs denoted as high voltage (HV) and low voltage (LV) owing to the voltage levels they are processing. The HV pair is composed of transistor M1-M2 and the LV pair is composed of transistors M3-M4. The current from the two differential pairs is summed by the folded cascode (M7-M8, M10-M15) followed by a second gain stage (M9, M16-M17), voltage follower M20 completes the feedback loop around the low voltage differential pair. The current mirrors M23-M28 copy the current through terminal $\mathrm{X}$ to the outputs Z1 and Z2. Current source M21-M22 adds an offset current $I_{\mathrm{off}}$ for bidirectional operation.

The HV differential pair is biased from the HV supply by means of the current mirror M29-M32 where the cascodes are based on DMOS transistors. To sustain high voltage the HV differential pair is cascoded by DMOS transistors M5M6. In order to not load differential pair or its biasing a separate circuit composed of M33-M38 is used to bias the gates. 
The M33-M35 form a differential pair with M33 and M34 averaging the input voltage.

The transfer of the voltage from the HV input pair to the node $X$ is given by the ratio of the transconductances of the two differential pairs, i.e.

$$
\alpha=\frac{g_{m}^{H V}}{g_{m}^{L V}}=\frac{g_{m}^{M 1}}{g_{m}^{M 3}} .
$$

The mismatch between the two differential pairs and their bias current will cause mismatch between the two transconductances. This together with the process variations of the internal resistor $R_{\text {copy }}$ will lead to the gain inaccuracy. Both of these effects can be trimmed by using the relation for $\alpha$ and trimming the transconductance of the HV stage. This is done by varying the bias current of the HV stage since to a first degree the transconductance is proportional to the bias current [12]. The bias current is set by the gain trimming DAC and mirrored by the HV current mirror M29-M32.

The gain trimming DAC is depicted in Fig. 4.

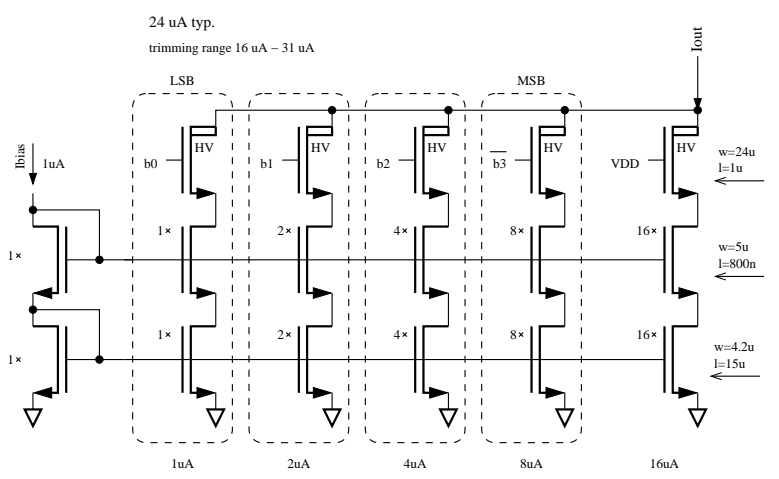

Fig. 4. Gain trimming DAC (dimensions apply for entire rows).

It is based on binary scaled current sources with added offset current. The typical current value is $24 \mu \mathrm{A}$ with a trimming range spanning from $16 \mu \mathrm{A}$ to $31 \mu \mathrm{A}$.

\section{Implementation and Results}

The proposed current sensor was realized in $0.35 \mu \mathrm{m}$ BCD technology from STMicroelectronics. The area of the current sensor is $0.387 \times 0.345 \mathrm{~mm}^{2}$ as can be seen in Fig. 5 . The LV part is supplied from an on-chip $3.3 \mathrm{~V}$ regulator whereas the HV part is supplied from the input voltage of the DC-DC converter which can go as high as $40 \mathrm{~V}$. For a typical input voltage of $12 \mathrm{~V}$ the circuit consumes $3.2 \mathrm{~mW}$.

Fig. 6 shows the simulated transfer characteristics for different trimming combinations. The gain trimming range is within $\pm 22 \%$ with a step about $2.8 \%$. This is sufficient to trim the process variation of the internal resistor $R_{\text {copy }}$ which is the major contributor of the gain variation.

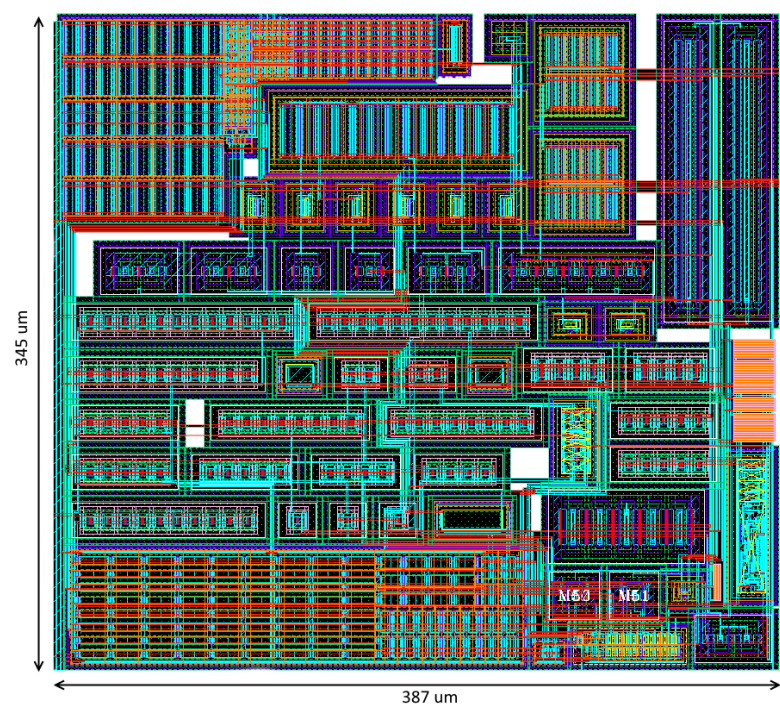

Fig. 5. Layout of the proposed circuit.

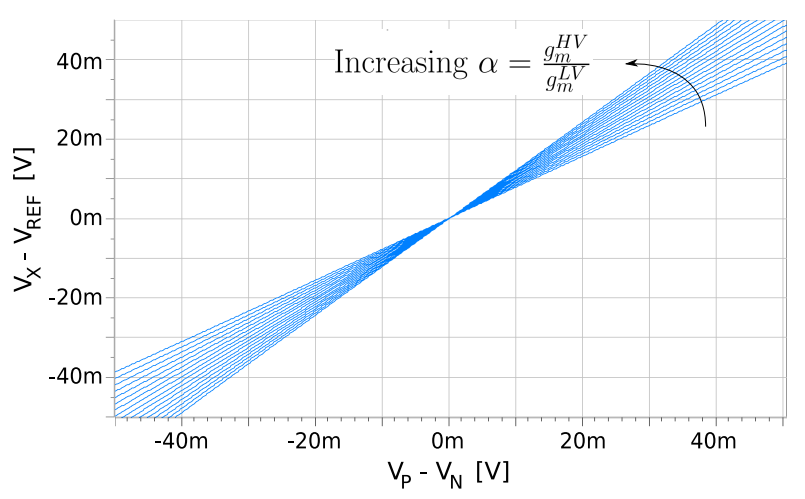

Fig. 6. Transfer characteristics.

Due to the nonlinear dependence of $g_{m}$ on $I_{D}$ the linearity of the sensor deteriorates with the trimming going further from the default value. This can be seen on the nonlinearity characteristics in Fig. 7. However in the given application the input differential voltage usually does not exceed $20 \mathrm{mV}$ for which the error caused by the nonlinear behaviour of the current sensor does not exceed $0.2 \%$.

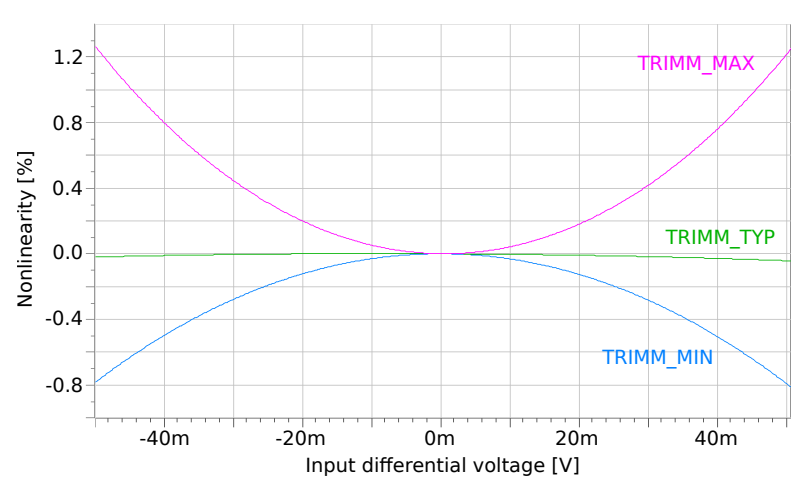

Fig. 7. Achieved nonlinearity $\left(I_{\text {sim }} / I_{\text {ideal }}-1\right)$ for different trimming steps.

The AC gain and phase response from the input terminal to the $\mathrm{X}$ node are in Fig. 8. The bandwidth reaches $50.9 \mathrm{MHz}$. 


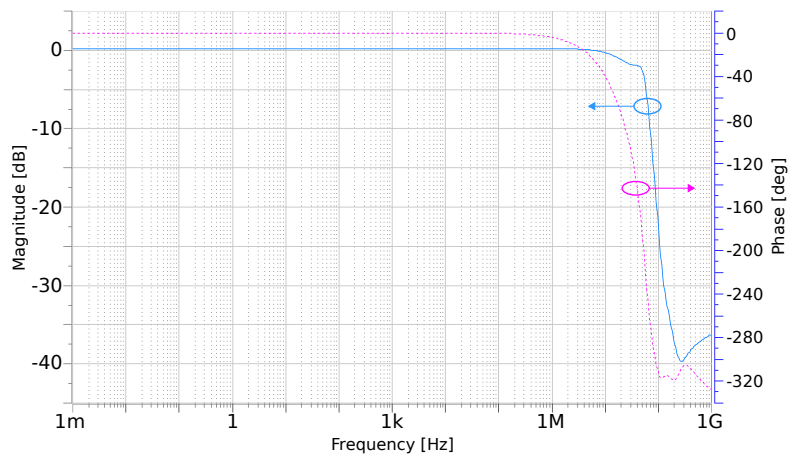

Fig. 8. Gain and phase characteristics of $V_{X} / V_{\text {sense }}$.

The proposed circuit was taped-out as part of a fourphase buck DC-DC converter whose application board is in Fig. 9. The output of the current sensor is used for overcurrent protection and for balancing the current evenly among all the phases. Although the current sensor could not be measured directly the operation can be verified by the balancing function of the system, as shown in Fig. 10. The waveforms labeled IL1, IL2 and IL4 are coil currents corresponding to three of the phases and are sensed by the described circuit as in Fig. 3. The same average currents of the phases show correct operation of the circuit.

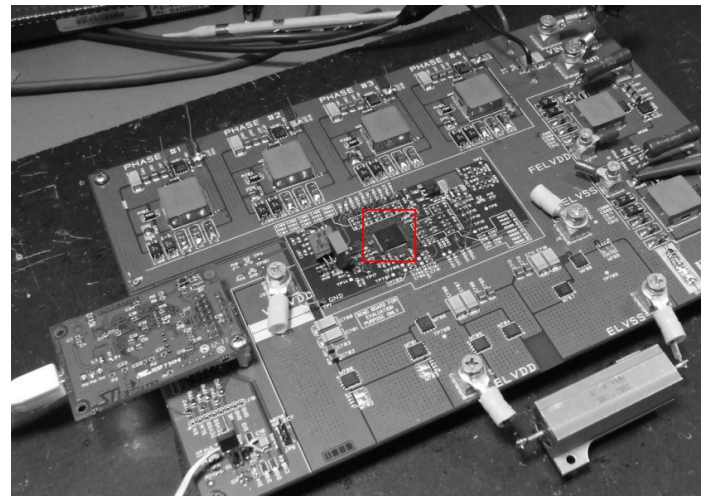

Fig. 9. Application board with the device (mark in square) featuring the proposed current sensor.

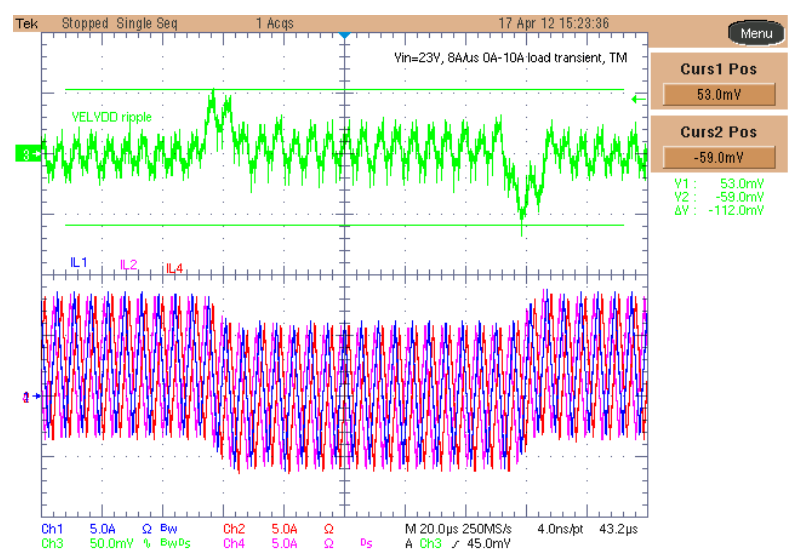

Fig. 10. Output voltage and current of three phases balanced with the help of the proposed sensor.

\section{Conclusion}

In this paper a new circuit for coil current sensing in high voltage DC-DC converters has been presented. A method for gain trimming based on transconductance variation of the input stage has been described.

The proposed solution has no static input current and the usage of high voltage components is limited to the input stage thereby reducing power consumption and silicon area. This is in contrast with traditional approaches, such as [7-9], where the operational amplifier has to be designed with both the input and output stages able to sustain high voltage. Another approach presented in [10] requires high voltage capacitors and the need for reset phase further complicates design and top-level integration. On the other hand, the presented sensor does not employ any special components but the standard CMOS and DMOS transistors present in any BCD type technology. Also the continuous operation simplifies integration with the rest of the application.

The circuit has been implemented in STMicroelectronics $0.35 \mu \mathrm{m}$ BCD technology and occupies area of $0.134 \mathrm{~mm}^{2}$. It has been integrated as part of a multi-phase DC-DC converter and its function has been verified. The proposed solution is suitable for DC-DC converters with external power MOS transistors where traditional sensors based on copy MOS transistors are not possible as one cannot effectively match integrated transistors with external ones from a different lot or possibly a different technology.

\section{Acknowledgments}

The authors would like to thank STMicroelectronics for design fabrication and measurements. The work has been supported by the grant No. SGS12/143/OHK3/2T/13 of the CTU in Prague.

\section{References}

[1] PATEL, A., FERDOWSI, M. Current sensing for automotive electronics - a survey. IEEE Transactions on Vehicular Technology, 2009, vol. 58, no. 8, p. 4108-4119. DOI: 10.1109/TVT.2009.2022081

[2] FORGHANI-ZADEH, H. P., RINCON-MORA, G. A. Currentsensing techniques for DC-DC converters. In The 2002 45th Midwest Symposium on Circuits and Systems (MWSCAS). 2002, vol. 2, p. II-577-II-580. DOI: 10.1109/MWSCAS.2002.1186927

[3] DU, M., LEE, H. An integrated speed- and accuracy-enhanced CMOS current sensor with dynamically biased shunt feedback for current-mode buck regulators. IEEE Transactions on Circuits and Systems I: Regular Papers, 2010, vol. 57, no. 10, p. 2804-2814. DOI: 10.1109/TCSI.2010.2046258

[4] LEE, C. F., MOK, K. T. A monolithic current-mode CMOS DCDC converter with on-chip current-sensing technique. IEEE Journal of Solid-State Circuits, 2004, vol. 39, no. 1, p. 3-14. DOI: 10.1109/JSSC.2003.820870 
[5] YUAN, B., LAI, X. On-chip CMOS current-sensing circuit for DCDC buck converter. Electronics Letters, 2009, vol. 45, no. 2, p. 102 103. DOI: 10.1049/el:20092855

[6] WANG, S. W., CHO, GYU-HA, CHO, GYU-HYEONG A highstability emulated absolute current hysteretic control single-inductor 5-output switching DC-DC converter with energy sharing and balancing. In IEEE International Solid-State Circuits Conference Digest of Technical Papers (ISSCC) 2012, p. 276-278. DOI: 10.1109/ISSCC.2012.6177002

[7] SUN, J., ZHOU, J., XU, M., LEE, F. C. A novel input-side current sensing method to achieve AVP for future VRs. IEEE Transactions on Power Electronics, 2006, vol. 21, no. 5, p. 1235-1242. DOI: 10.1109/TPEL.2006.880347

[8] HWU, K. I., CHEN, Y. H. Current sharing control strategy based on phase link. IEEE Transactions on Industrial Electronics, 2012, vol. 59, no. 2, p. 701-713. DOI: 10.1109/TIE.2011.2151820

[9] SIMON-MUELA, A., PETIBON, S., ALONSO, C., CHAPTAL, J.-L. Practical implementation of a high-frequency current-sense technique for VRM. IEEE Transactions on Industrial Electronics, 2008, vol. 55, no. 9, p. 3221-3230. DOI: 10.1109/TIE.2008.927968

[10] WICHT, B., KULHARNI, S. P., HERZER, S., NEIDHARDT, J. Switched Mode Power Supply with Current Sensing, US Patent 8,203,323. June 19, 2012.

[11] CHIU, W., LIU, S.-I., TSAO, H.-W., CHEN, J.-J. CMOS differential difference current conveyors and their applications. IEE Proceedings -Circuits, Devices and Systems, 1996, vol. 143, no. 2, p. 91-96. DOI: 10.1049/ip-cds: 19960223
[12] ENZ, C. C., KRUMMENACHER, F., VITTOZ, E. A. An analytical MOS transistor model valid in all regions of operation and dedicated to low-voltage and low-current applications. Analog Integrated Circuits and Signal Processing, 1995, vol. 8, no. 1, p. 83-114. DOI: 10.1007/BF01239381

\section{About the Authors...}

Martin Ď̌íNOVSKÝ was born in 1985. He received his Master's degree from the Czech Technical University in Prague and is currently studying towards Ph.D. at the Department of Circuit Theory. He is with STMicroelectronics since 2010 working as a senior design engineer. His research interests include analog and mixed-signal circuits, IP modeling and verification methodologies.

Jiří HOSPODKA was born in 1967. He received his Master's and Ph.D. degrees from the Czech Technical University in Prague in 1991 and 1995, respectively. Since 2007 he has been working as associate professor at the Department of Circuit Theory at the same university. Research interests: circuit theory, analogue electronics, filter design, switchedcapacitor, and switched-current circuits. 